\title{
A scatter diagram for displaying the response of sensory neurons to sinusoidal stimulation
}

\author{
T. C. T. YIN* AND W. J. WILLIAMS
}

Bioelectrical Sciences Laboratory, Department of Electrical and Computer Engineering, University of Michigan, Ann Arbor, Mich. 48104 (U.S.A.)

(Accepted February 21st, 1974)

The quantitative study of the activity of single neurons in sensory systems has been a subject of increasing interest in recent years. As a result there are a number of statistical tools suitable for analyzing the patterns of neuronal activity from both receptors and higher-order cells ${ }^{1}$. This has contributed to a greater understanding of the detailed mechanisms of sensory transduction and coding in these peripheral and central processes. Since the response of sensory neurons is usually characterized by the average frequency of discharge, a number of good quantitative techniques have found application in the analysis of tonic responses. However, very little quantitative analysis of phasic neurons has been carried out. Most investigators simply note their existence. This communication describes a convenient display for characterizing the 'frequency response' of a phasic sensory cell.

The technique developed out of the use of a swept frequency technique for extracting transfer functions of neural systems ${ }^{6}$. Briefly the technique utilizes a swept frequency sine wave stimulus that is then correlated with the neural output. If a describing function analysis is used, the system transfer function can be found regardless of whether the response is tonic or phasic, i.e. whether or not there is a carrier frequency.

In addition to the transfer function of the sensory system, it is often of interest to display the occurrence of pulses relative to the phase of the input signal. To do this the swept frequency data can be processed in a manner that is similar to the standard cycle histograms ${ }^{4}$ often employed with discrete sine wave inputs. A program was written to implement the display on a small laboratory computer (Hewlett-Packard 2115a). Phase angle is the abscissa and input modulation frequency is the ordinate. The beam of an oscilloscope is driven by the $\mathrm{D} / \mathrm{A}$ converter and follows the instantaneous frequency and phase of the stimulus. The beam is brightened only when a spike occurs. The display is essentially a binary matrix: the columns correspond to the intervals of phase (in our case, $5^{\circ}$ ) from $0^{\circ}$ to $360^{\circ}$ and each row is one period of the

\footnotetext{
* Present address: Laboratory of Neurobiology, 4234 Ridge Lea, State University of New York, Buffalo, N.Y. 14226, U.S.A.
} 
swept frequency input. Each pulse causes a point to be brightened at the appropriate phase angle (along the abscissa) and at the particular modulation frequency (along the ordinate) corresponding to the period of the swept frequency in which it occurred.

The $n$ successive periods of the input swept frequency sine waves are calculated by measuring the times between successive positive-going zero-crossings. A spike occurring within any period $T_{j}$ produces a spot in row $j$ and in the column corresponding to the proper phase relative to the input.

The ordinate, then, plots the number $\mathrm{j}$ of the cycle in the swept input and each cycle is related to a particular frequency as the reciprocal of the period. We have used the Hewlett-Packard 3305 log sweep signal generator to generate the swept sine waves. The unique aspect of the display is that for logarithmic sweeps with suitably long sweep times the relationship between $j$ and frequency is linear? Thus, for log sweeps the ordinate is a linear frequency axis over the bandwidth of the sweep, i.e. over the limits $f_{\min }$ and $f_{\max }$ where $f_{\min }=1 / T_{1}$ and $f_{\max }=1 / T_{n}$. Fig. 1A shows the display that is obtained for a simulation in which a tonic pulse train with a constant carrier is frequency modulated by a swept sinusoidal input. The pulse train was produced by modulating a voltage-controlled pulse generator with the $\log$ sweep. In Fig. IB the
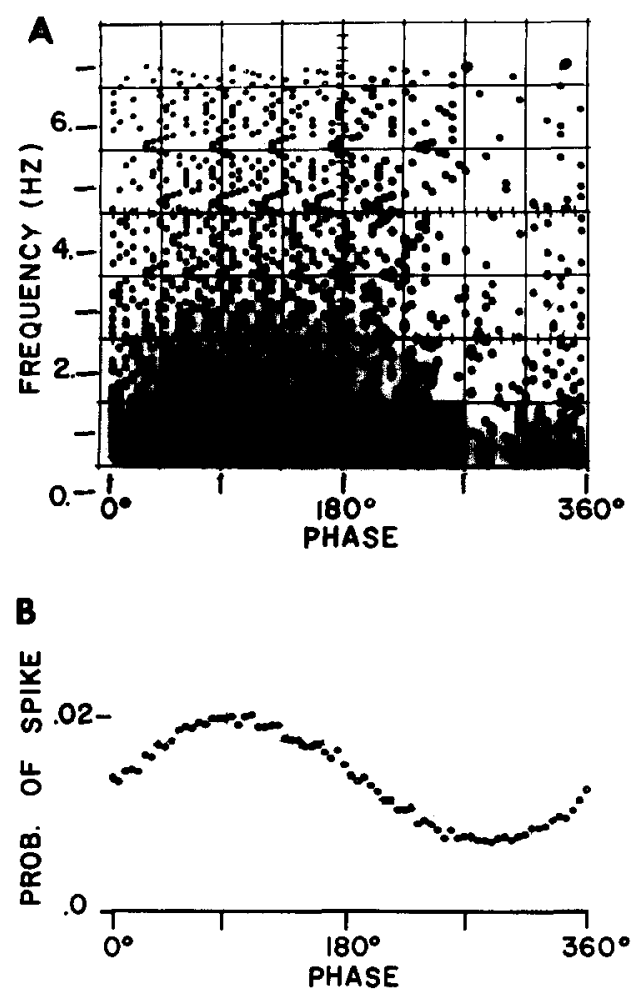

Fig. 1. A: scatter diagram for a simulated tonic neuron. The pulse train was generated by modulating a voltage-controlled pulse generator with the swept frequency $(0.3-7 \mathrm{~Hz})$ waveform. Average pulse rate was 30 pulses/sec. B: composite 'cycle histogram' of the swept frequency input obtained by binning the columns in A. This illustrates the reconstruction of the input sine wave. 


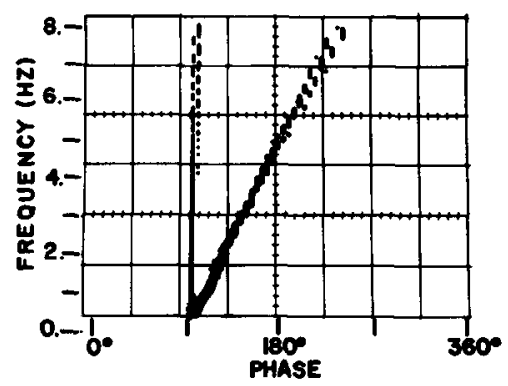

Fig. 2. Scatter diagram for a simulated phase-locked neuron. Each period of the swept frequency input waveform generated a pair of pulses such that the first pulse occurred at $90^{\circ}$ phase angle and the second pulse always followed the first by $50 \mathrm{msec}$.

columns have been binned. Since the phase of the response is the same at all frequencies for this simulation, the original sine wave is reconstructed in Fig. 1B.

A common characteristic of neural systems that are subjected to periodic stimuli such as sine waves is a phase-locking of the output spikes to the input waveform ${ }^{3,4}$. For discrete sinusoids this phase locking is manifested as a peak in the cycle histograms. Rescigno et al. ${ }^{2}$ have shown that this behavior can be predicted by simple neural models stimulated with cyclic inputs. The scatter diagram described above is especially suited to detecting the onset and frequency dependency in this phase-locked characteristic.

A phase-locked response can be easily detected in the scatter diagram as a distinctive column of dots. The frequency at which this begins is given by the lowest point of the column. In addition since the axes are linear for logarithmic sweeps, a constant time delay (or latency) associated with this phase-locked response will produce a straight line of dots with a positive slope. The value of the delay is given by the reciprocal of the slope of the line. Fig. 2 gives the results of a simulation that illustrates these points. A pair of pulses was generated by a pulse generator driven by the swept frequency input such that the first pulse always occurred at the peak of the input sine wave (phase $=90^{\circ}$ ) and the second pulse always followed the first but with a delay of 50 msec.

We have used this display in our laboratory in the study of the knee joint receptor system in the cat ${ }^{5,7}$. These receptors are sensitive to position and movement of the joint, in this case to the angle of the tibia with respect to the femur. Fig. 3 shows the response of neurons in the VPL of the thalamus that are sensitive to knee joint movement. In Fig. 3A the cell exhibited a spontaneous discharge and the input sinusoid caused a response that occurred between $0^{\circ}$ and $90^{\circ}$ phase at the higher frequencies. There was a slight phase lag at the higher frequencies as evidenced by the slight slope in the response. Fig. 3B shows another cell that exhibited a very low rate of spontaneous discharge and became phase-locked near $300^{\circ}$ phase at very low input frequencies. In addition, the phase at which the cell responded showed a gradual lag, as evidenced by the slope of the line of dots in the scatter diagram, so that the response phase changed from $+360^{\circ}$ to $0^{\circ}$ around $4 \mathrm{~Hz}$. Since the line was linear, it implied that the lag was 

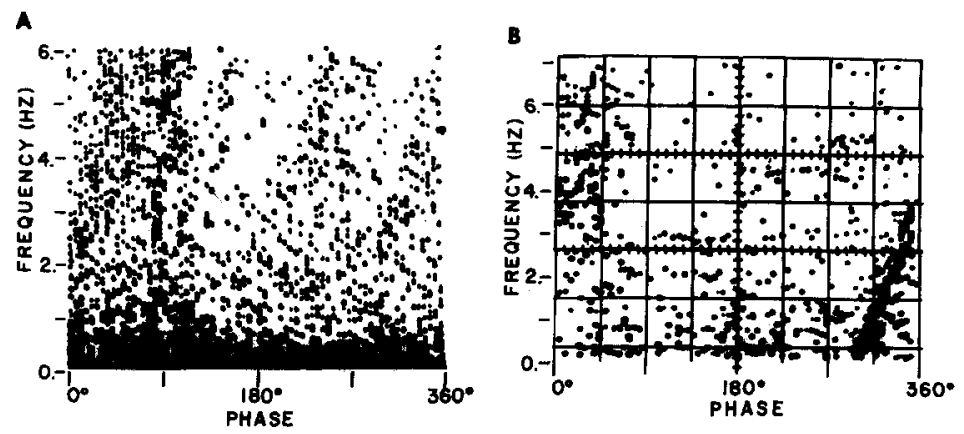

Fig. 3. Scatter diagrams for phasic knee joint afferents in the VPL of cats. A : neuron was recorded in an unanesthetized cat while the joint was being articulated by a swept frequency waveform $(0.1-6 \mathrm{~Hz})$. B: neuron was recorded in a pentobarbital-anesthetized cat. Pronounced phase-locking was evident near $300^{\circ}$ phase even at very low frequencies.

due to a time delay factor. However, the delay predicted by the slope of the line ( 51 $\mathrm{msec}$ ) was much greater than the conduction delays $(23 \mathrm{msec})$ obtained by measuring the latency to step inputs applied to the knee joint ${ }^{7}$. The additional delay is evidence of possible internal feedback loops. This is one example of the utility of this display. Other possible uses for this display are studies of the neural basis of flicker fusion or any studies of the frequency dependence of phase-locking in sensory cells to periodic inputs.

For stochastic systems where time averaging is needed to extract a reliable response, the total sweep time between the high and low frequency limits can be made as long as necessary without changing the bandwidth in order to get a greater ratio of number of cycles to frequency band. Since all of this information can be obtained from one sweep (150 sec gives about 250 cycles between 0.1 and $7 \mathrm{~Hz}$ for the logarithmic sweep), this represents a considerable saving of time over that required in using conventional discrete sine waves. Since the change in the frequency is small from cycle to cycle, the deviation of the stimulus sinusoid from a sinusoid of fixed frequency is very small over a single cycle. However, the slight change in the frequency of the stimulus sinusoid may prevent the frequency entrainment of spikes sometimes observed when a series of identical sinusoidal stimuli is applied. Frequency entrainment of spike trains by a constant frequency stimulus is not likely in a realistic physiological setting since a stimulus of the required purity would be extremely rare if not nonexistent. Phase locking on a cycle-by-cycle basis will still occur, however, and is amenable to analysis by this technique.

The analysis technique described in this paper does not require the complexity of the digital computer for its realization. A simple analog circuit could undoubtedly accomplish the same end.

Supported by U.S. Public Health Service Grant NS 08470. 
1 Perkel, D. H., Gerstern, G. L., And Moore, G. P., Neuronal spike trains and stochastic point processes. I. The single spike train, Biophys. J., 7 (1967) 391-418.

2 Rescigno, A., Stein, R. B., Purple, R. L., and Poppele, R. E., A neuronal model for the discharge patterns produced by cyclic inputs, Bull. Math. Biophys., 32 (1970) 337-353.

3 Rose, J. E., Brugge, J. F., Anderson, D. J., And Hind, J. E., Phase-locked response to low-frequency tones in single auditory nerve fibers of the squirrel monkey, J. Neurophysiol., 30 (1967) 769-793.

4 Talbot, W. H., Darian-Smith, I., Kornhuber, H. H., and Mountcastle, V. B., The sense of flutter-vibration: comparison of the human capacity with response patterns of mechanoreceptive afferents from the monkey hand, J. Neurophysiol., 31 (1968) 301-334.

5 Williams, W. J., BeMent, S. L., Yin, T. C. T., ANd MCCAll, JR., W. D., Nucleus gracilis responses to knee joint motion: a frequency response study, Brain Research, 64 (1973) 123-140.

6 Williams, W. J., Gesink, J. W., And Stern, M. M., Biological system transfer function extraction using swept frequency and correlation techniques, Med. biol. Engng., 10 (1972) 609-620.

7 Yin, T. C. T., Dynamic Response and Transfer Characteristics of Knee Joint Afferents in Somatosensory Thalamus of the Cat, Ph.D. Thesis, Univ. of Michigan, 1973. 